\title{
DIFICULTADES EN LA ENSEÑANZA-APRENDIZAJE DE LOS PROBLEMAS DE FÍSICA Y QUÍMICA. I. OPINIONES DEL ALUMNO
}

\author{
OÑORBE DE TORRE, A. ${ }^{1}$ y SÁNCHEZ JIMÉNEZ, J.M. ${ }^{2}$ \\ I IB Emilia Pardo Bazán. \\ 2 Departamento de Química Física. Universidad de Alcalá de Henares. Madrid.
}

\begin{abstract}
SUMMARY
Analysing the ideas with which teachers and pupils confront problems in physics and chemistry. The starting point for this analysis is the compilation of the difficulties and causes of failure involved in solving problems raised during a didactic investigation of these subjects.
\end{abstract}

This part shows the results obtained in relation to the pupils. They show their opinions on the difficulties they came across, their evaluations, relationships between these and other groups of students showing similar characteristics. Another paper will be dedicated to the teacher's opinions and a comparison between both groups.

\section{INTRODUCCIÓN}

La utilización de problemas en Ia educación científica ha sido siempre una parte muy importante del trabajo realizado por profesores y alumnos. La sistematización de los estudios realizados en resolución de problemas de física y química presenta grandes dificultades debido a que las concepciones iniciales tanto del propio problema como de su proceso de resolución difieren notablemente. Diversos autores aportan en sus trabajos visiones generales del tema y de su actual desarrollo (Reif, 1981; Fuller, 1982; Frazer, 1982; Kempa, 1983; Garrett, 1986; Gil Pérez y Martínez Torregrosa, 1988).

Los resultados de las investigaciones relevantes sobre dificultades en la resolucion de problemas aparecen relacionados con factores muy diversos. Estudios de dificultades asociados a la comprension del enunciado: forma de su descripción (Reif, 1983), lenguaje y organización de la información (Long, 1991), cantidad de términos científicos (Merzyn, 1987), información redundante o superflua (Johnstone y Kellet, 1980; Kempa, 1986). Dificultades asociadas a conocimientos de los alumnos: falta de conocimientos específicos del tema que alegan los profesores (Reyes y Furio, 1988), conceptuales o procedimentales (Stewart, 1982), fallo en la utilización de conocimientos conocidos (Mc Cabe, 1977) o en su conexión (Gorodestky y Ron, 1980). Dificultades asociadas a estrategias de resolución: utilización de técnicas adecuadas (Lythcott, 1990), utilización de reglas y modelos fijos (Dumas-Carré, 1987), etapas o subproblemas (Frazer, 1984; Bodner, 1987). Dificultades asociadas a estructuras cognitivas y características personales: fallos de memoria (Kempa, 1986), comparación entre la M.demanda y la M.capacidad (Opdenacker, 1990), alumnos dependientes o independientes de campo (Niaz, 1989), autoconfianza y falta de interés (Garrett, 1986).

Los trabajos sobre dificultades de la resolución de problemas se refieren, en general, a aspectos muy diversos deI alumno; pero no suelen tener en cuenta las interacciones que, en el proceso de enseñanza-aprendizaje, corresponden al pensamiento de profesores y alumnos. 
Entre las ideas preconcebidas que el profesor de física y química lleva a sus clases suele citarse el alto porcentaje, admitido como natural, de fracaso del aprendizaje de resolución de problemas, relacionado con factores externos de la propia enseñanza: falta de capacidad intelectual de los alumnos, su falta de interés, trabajo, preparación anterior, etc. Estas concepciones están extendidas a gran número de profesores, independientemente de su origen y formación (Garrett y Sánchez, 1992).

Especialistas de didáctica de las ciencias experimentales han señalado la importancia de conocer el pensamiento espontáneo docente, que marca el enfoque metodológico de la enseñanza impartida (Shuell, 1987; Hewson, 1988). En el campo de resolución de problemas, algún estudio señala la importancia de la reflexión sobre el pensamiento propio del profesor (GiI, 1991), pero una línea de investigación, de gran interés y no suficientemente tratada, es la de mostrar las ideas previas de profesores y alumnos sobre resolución de problemas de fúsica y química y establecer una comparación entre ellas. Con este objetivo, nuestro trabajo detecta las opiniones previas que profesores y alumnos tienen sobre las dificuliades de la resolución de problemas, establece relaciones entre las variables, caracteriza grupos en función de las ideas que tienen sobre resolución de problemas y hace un estudio comparativo entre las opiniones de profesores y alumnos.

\section{DISEÑO EXPERIMENTAL}

El estudio de las investigaciones permitieron obtener una serie previa de posibles dificultades. A partir de ella se confeccions un conjunto de treinta ítems que se entregó a quince profesores experimentados de física y química de los distintos niveles de enseñanza (jueces profesionales). Se les solicitó su opinión sobre los f́tems propuestos, suimportancia, lenguaje utilizado, claridad,etc., así como una clasificación dentro de los siguientes apartados: referidos al que enseña, al que aprende, a conocimientos (conceptuales y procedimentales) y al entorno de la enseñanza y factores externos.

Con su opinión se confeccionaron dos tipos de encuestas, dirigidas a profesores y alumnos, similares en su contenido. Se contrastaron con un grupo de prueba (profesores y alumnos) que, después de contestar, fueron entrevistados para analizar la posible influencia de la redacción de los f́tems, el orden de presentación y la comprensión de las instrucciones. Se añadio, para los alumnos, una petición de definición sobre lo que consideraban un problema de física y química y una valoración cualitativa de la dificultad, mayor o menor, de la teoría o de los problemas. A los profesores se les pregunt 6 qué porcentaje de sus alumnos creía que fracasaban en Ia teoría y en los problemas. A los profesores se les pidió una valoración de $I$ a 10 para cada ítem mientras que para Ios alumnos se estimó más adecuada la valoración entre 0 y 5 ( máxima dificultad 5), ya que se observó que la calificación de uno a diez tendían a utilizarla como una ordenación y no una valoración.
Todos los datos cuantitativos obtenidos han sido registrados en DBase. El análisis estadístico se realizó con los siguientes programas: BMDP5D, de descriptiva univariante; $\mathrm{BMDP} 3 \mathrm{D}$ y $\mathrm{BMDP7D}$, de tratamiento estadístico comparativo, aplicados a profesores y alumnos respectivamente; BMDP4M, de análisis factorial y SPAD.N, para clasificación de sujetos.

En este artículo se presentan Ios resulfados obtenidos para los alumnos. La encuesta de opinión utilizada aparece en el anexo I, donde también se incluyen las variables utilizadas con el número y nombre asignado a cada una de ellas para las tablas y gráficas.

La encuesta fue contestada, válidamente, por 419 alumnos, fundamentalmente de bachillerato y $\operatorname{COU}(71,1 \%)$, estudiantes ingleses $(20,3 \%)$ con el fin de comparar respuestas con las provenientes de otro sistema educativo, y estudiantes del último curso de EU de Formación del Profesorado de Ciencias $(8,6 \%)$, cuyo pensamiento sobre las dificultades de resolución de problemas incidirá en su futura práctica docente.

\section{ANÁLISIS DE LOS RESULTADOS}

Para la variable «¿Qué es más difícil, la teoria o los problemas?», responden 331 sujetos (todos los españoles) y se encuentra una clara mayoría $(69,8 \%)$ de alumnos que considera más difícil los problemas, frente a un

Gráfica I

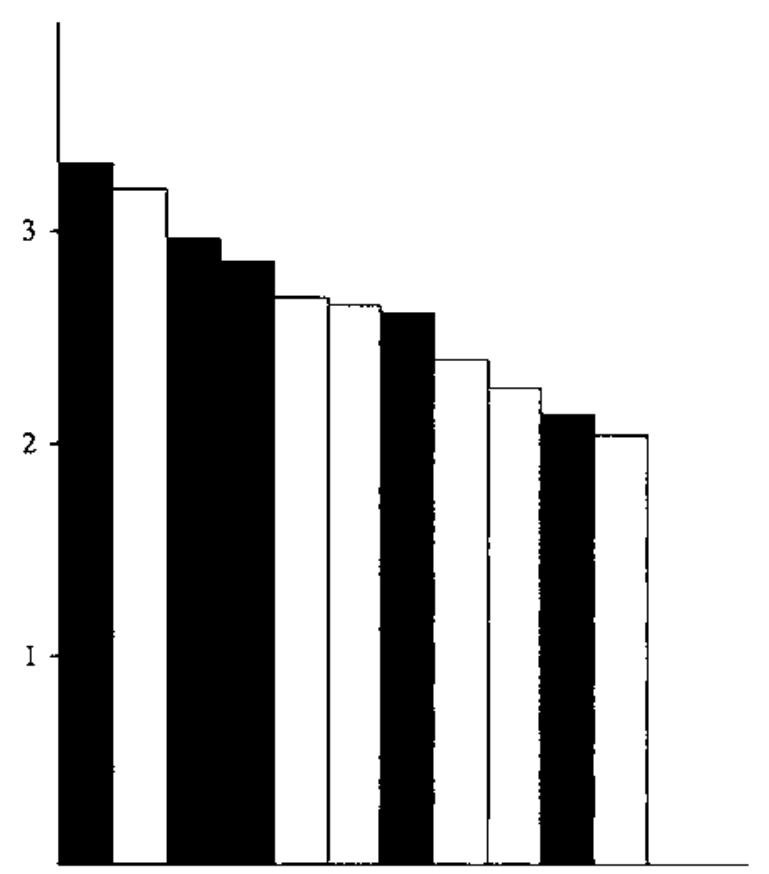

Ap Cm En Co Cn Tr Me Cal Cla Int Cfi 
$19,9 \%$ que considera más diff́cil la teoría y un $10,3 \%$ de igual dificultad.

A partir de los valores medios otorgados a las dificultades (Gráfica I) y de la distribución de estos valores, se establecen los siguientes grupos de dificultades:

1) Aplicación-teoría a problemas y camino para resolverlo.

Son las dificultades que obtienen valores más altos. Podrían unirse bajo el título de «estrategias de resolución” o de conocimiento procedimental.

2) No se entiende y son complicados.

Ambas observaciones se refieren al propio problema antes de comenzar a resolverlo, es decir a su lenguaje y planteamiento inicial.

3) Falta de conocimientos, de trabajo y fallos de memoria.

Referentes al propio alumno, en sus conocimientos teoricos conceptuales, de conocimiento declarativo, explicado por la falta de trabajo personal.

4) Fállo en los cálculos, no se entienden en clase, falta de interés y de confianza en sí mismo.

Es el grupo de menor puntuación y más disperso. Se refieren a dificultades externas, cálculo y profesor, y a características personales.

Se han calculado los valores medios y desviaciones estándar para cada nivel de enseñanza en los f́tems de dificultades. El orden, de mayor a menor, en las puntuaciones medias para los distintos niveles es:

Nivel 1 (Ingleses): Memoria, Trabajo, Aplicación, Complicación, Camino, Entendimiento, Conocimiento, Confianza, Interés, Cálculo, Clase.

Nivel 2 ( $2^{\circ}$ de BUP): Camino, Aplicación, Entendimiento, Complicación, Cálculo, Memoria, Trabajo, Interés, Clase, Conocimiento, Confianza.

Nivel 3 ( $3^{\circ}$ de BUP): Camino, Aplicación, Trabajo, Complicación, Conocimiento, Entendimiento, Memoria, Clase, Interés, Confianza, Cálculo.

Nivel 4 (COU): Aplicación, Camino, Entendimiento, Complicación, Conocimiento, Trabajo, Cálculo, Memoria, Clase, Confianza, Interés.

Nivel 5 (E.U.FORM): Aplicación, Entendimiento, Camino, Complicación, Conocimiento, Memoria, Clase, Trabajo, Cálculo, Interés, Confianza.

La ordenación anterior muestra la escala de importancia de las dificultades para cada curso o nivel. En un primer análisis de estos resultados se pueden hacer algunas consideraciones. El ítem «aplicación de la téoría» crece claramente con el nivel y en cambio decrece la valoración de los ítems «falta de interés y la confianza en sí mismos». El nivel de $2^{\circ}$ de BUP presenta una alta significatividad para su inferior valoración dada al ítem «falta de conocimiento». El grupo de estudiantes ingleses mantiene opiniones claramente distintas a las de otros niveles, con alta significatividad en estas comparaciones.

Se efectuó el análisis factorial de los valores de las variables 4 a 14, que corresponde al estudio de correlacion entre las respuestas dadas por un mismo sujeto. Se aplicó a la muestra total de alumnos, sin distinción entre niveles. Aparecen cinco factores de correlación:

\begin{tabular}{lllll}
\hline Factor 1 & Factor 2 & Factor 3 & Factor 4 & Factor 5 \\
& & & & \\
Trabajo & Aplic. & Mem. & Confi. & Cálcu. \\
Interés & Camino & Compli. & Enten. & $\ldots \ldots \ldots$ \\
Conoc. & $\ldots \ldots \ldots .$. & Clase & $\ldots \ldots \ldots$ & Camino \\
Enten. & Clase & & (r)Conoc. &
\end{tabular}

Nota: Las zonas de puntos separan aquellos items con los que también hay alguna relación pero ésta es poco significativa.

Una primera caracterización de estos factores sería:

Factor I: El alumno responsable. El esfuerzo propio: su trabajo, interés y conocimientos conceptuales.

Factor 2: Estrategias de resolución. Conocimiento procedimental.

Factor 3: Dificultades externas y profesor. Fallos de memoria, complicación de los problemas y no entender en clase.

Factor 4: El alumno no responsable. Duda de su capacidad, no cree poder resolver el problema y no entiende el enunciado ni los que se hacen en clase.

Factor 5: Falla el cálculo. Prácticamente independiente de otras variables aunque con la falta de conocimientos presenta una pequeña relación negativa. Cree saber el tema pero se equivoca al operar.

El tratamiento estadístico de Ios datos para dividir el conjunto de la muestra en grupos de sujetos que presentan semejanzas en sus respuestas a las dificuitades permite obtener particiones. Las más significativas corresponden a dos clases A y B y a su división en dos nuevos grupos cada una (denominados 1 y 2,3 y 4 ).

Cuadro I

\begin{tabular}{|c|c|c|}
\hline $\begin{array}{c}\text { Clase A } \\
245 \text { alumnos }(58,6 \%)\end{array}$ & $\begin{array}{c}\text { Grupo 1 } \\
152 \text { alumnos }(36,4 \%)\end{array}$ & $\begin{array}{c}\text { Grupo 2 } \\
93 \text { alumnos (22,2\%) }\end{array}$ \\
\hline $\begin{array}{c}\text { Clase B } \\
173 \text { alumnos (41,4\%) }\end{array}$ & $\begin{array}{c}\text { Grupo 3 } \\
67 \text { alumnos (16\%) }\end{array}$ & $\begin{array}{c}\text { Grupo 4 } \\
106 \text { alumnos (25,4\%) }\end{array}$ \\
\hline
\end{tabular}


Cuadro II

[Fracaso P.

ALUMNOS: grupos A y B

La clase A presenta, en general, valores bajos para todos losítems, mientras que en la B estos valores son altos. Se exceptúa la variable «Calcu» que no se relaciona con los otros; este mismo resultado ya se había detectado en el análisis factorial.

El cuadro If resume la clasificación en grupos de los alumnos indicando las principales características de cada grupo.

El grupo $I$ es de valores bajos en general y presenta como características principales el valor medio de los ítems APLIC y ENTEN ( mucho menor que otros grupos), alto en CALCU, valor medio en MEM. Grupo Cálculo. Alumno/no responsable.

El grupo 2, también de valores bajos, acentúa esta tendencia en MEM y CONFI siendo altos APLIC y CAMINO. EI ítem CALCU es bajo y se encuentra en la media general el item ENTEN. Grupo Procedimiento.

El grupo 3 presenta valores muy altos en los ítems falta de TRAB, de INTER y de CONOC siendo normal su media en COMPLI y baja en CALCU. Grupo Alumno/ responsable.
El grupo 4 tiene muy altos los valores correspondientes a COMPLI y CLASE y bajos los de TRAB e INTER, siendo normal el valor CALCU. Grupo enseñanza (profesor).

\section{CONCLUSIONES}

Los estudiantes asignan la máxima dificultad a los procedimientos de resolución y a la incomprensión de los enunciados. Admiten moderamente su responsabilidad por falta de trabajo y atribuyen escasa influencia en su fracaso a Ios errores de cálculo o a no entenderlos en clase.

Los alumnos vinculan entre si las dificultades relativas a:

- Procedimientos de resolución.

- Su falta de trabajo e interés.

- La falta de confianza en sí mismos con la comprensión del enunciado. 
- La enseñanza recibida con la excesiva complicación de los problemas.

- Los errores de cálculo y fallos de memoria aparecen como variables independientes.

Los alumnos se clasifican en cuatro grupos por el pensamiento con que se enfrentan a su aprendizaje.

Los conjuntos tipificados son :

- Alumno/ no responsable: Valoraciones bajas en todas las dificultades, excepto en cálculo.

- Procedimientos: considera la falta de conocimientos procedimentales como principal causa de su fracaso.

\section{REFERENCIAS BIBLIOGRÁFICAS}

BODNER, G.M. (1987). The Role of Algorithm in Teaching Problem Solving. Journal of Chemical Education, 64(6), pp. 513-514.

DUMAS-CARRE, A. y LARCHER C. (1987). The stepping stones of learning and evaluation. International Journal of Science Education, 9(1), pp. 93-104.

FRAZER, M.J. (1982). Solving Chemical Problems. Chemical Society Revue, 11(2), pp. 171-190.

FULLER, R.G. (1982). Solving Phisycs Problems. How do we do it? Physics today. Sept., pp. 43-47.

GARRETTR, M. (1986). Problem-Solving in Science Education. Studies in Science Education, 13, pp. 70-95.

GARRE'T, R.M. y SÁNCHEZ JIMÉNEZ, J.M. (1992). A comparison of Spanish and English Teachers' views of Problem Solving. Comparative Education, 28(3), pp. 269280 .

GIL PÉREZ, D., MARTÍNEZ TORREGROSA, J. y SENENT PÉREZ, F. (1988). El fracaso en la resolución de problemas de física: una investigación orientada por nuevos supuestos. Enseñanza de las Ciencias, 6(2), pp. 131-146.

GIL, D. (1991). ¿Qué han de saber y saber hacer los profesores de ciencias? Enseñanza de las Ciencias, 9(1), pp. 69-77.

GORODETSKY, M. y RON, H. (1980). Use of concept, profile Analy sis to Identify difficulties in Solving Science Problems. Science Education, 64(5), pp. 671-678.

HEWSON, P.W. y HEWSON, M.G. (1987). Science teachers'conceptions of teaching: Implications for teacher education. International Journal of Science Education, 9(4), pp. $425-440$.

IOHNSTONE, A.H. y KELLET, N.C. (1980). Learning difficulties in school science-towards a working hypothesis. European Journal of Science Education, 2(2), pp. 175-181.

KEMPA, R.F. y NICHOLLS, C.E. (1983). Problem solving ability and cognitive structure an explorator investigation. European Journal of Science Education, 5(92), pp.171-184.
- Alumno / responsable: Asumen en mayor proporción su propia responsabilidad por falta de trabajo, interés y conocimientos teóricos.

- Profesor/enseñanza: Asocian más el fracaso a la enseñanza y al profesor.

Los resultados obtenidos ofrecen pautas organizadas para la reflexión acerca de las ideas previas de tos alumnos relacionadas con las dificultades del aprendizaje en la resolución de problemas de física y química. En general, entre los estudiantes de una clase se encontrarán estadisticamente representantes de los cuatro grupos descritos. La metodología đe enseñanza empleada habrá de tener presente las dificultades supuestas por los alumnos si se quiere incidir positivamente en el proceso de aprendizaje de la «resolución de problemas».

KEMPA, R.F. (1986). Resolucion de problemas de química y estructura cognitiva. Enseñanza de las Ciencias, 4(2), pp. 99-110.

LONG, R.R. (1991). Readability for Science, some factor which may affect the students'understanding of worksheets, etc. School Science Review, 73(262), pp. 21-33

LYTHCOTT, J. (1990). Problem Solving and Requisite Knowledge of Chemistry. Joumal of Chemical Education, $7(3)$, pp. 248-252.

Mc CABE, R. (1977). A study of students Difficulties in Solving Chemical Problems, East Anglia, julio, 198.

MERZYN, O. (1987). The language of school Science. International Journal of Science Education, 9(4), pp. 483.489

NIAZ, M. (1989). The relation between M-Demand, Algorithms and Problem solving. A neo-Piagetian Analysis. Journal of Chemical Education, 66 (5), pp. 422-424.

OPDENACKER, C. y FIERENS, $\mathrm{H}$. et al, (1990). Academic performance in solving chemistry problems related to student wotking memory capacity. International Journal of Science Education, 12(2), pp. 177-185.

REIF, F. (1983). How can Chemists Teach problem-solving? Suggestions derived from studies of Cognitive Processes. Journal of Chemical Education, 60(11), pp. 948-953.

REIF, F. (198I). Teaching problem solving - A scientific approach. The Physics Teacher, 19(5), pp. 310-316.

REYES, V. y FURIÓ, C. (1988). Opinión de los profesores sobre las causas del fracaso escolar en la resolución de problemas de química. III Jornadas para la Renovacion Metodológica de la Enseñanza Media y C.S. Bilbao: ICE Universidad del País Vasco.

SHUELL, T.J. (1987). Cognitive psychology and conceptual change: implications for teaching science. Science Education, 71(2), pp. 239-250.

STEWART, J. (I982). Two aspects of Meaningful Problem Solving in Science. Science Education, 66(5), pp. 731-749. 
ANEXO I

\begin{tabular}{|c|c|c|}
\hline VARIABLE & NOMBRE y NÚMERO & RANGO \\
\hline Nivel educativo & Nivel, 2 & $\begin{array}{l}\left.\left.1 \text { (Ingleses), } 2 \text { (BUP } 2^{\circ}\right), 3 \text { (BUP } 3^{\circ}\right), \\
4 \text { (COU), } 5 \text { (Esc. univ.) }\end{array}$ \\
\hline Más d:fícil teoría o problemas & Teor-Pro, 3 & 0 (Igual) 1 (Teor.) 2 (Prob.) \\
\hline Aplicacion de la teoría & Aplic, 4 & Cuantitativo - de 0 a 5 \\
\hline Incomprensión del enunciado & Enten, 5 & Cuantitativo - de 0 a 5 \\
\hline Falta de conocimiento & Conoc, 6 & Ídem \\
\hline Falta de memoria & Memo, 7 & Ídem \\
\hline Falta de trabajo & Traba, 8 & Ídem \\
\hline Falta de interés & Inter, 9 & Ídem \\
\hline Demasiado complicado & Compli, 10 & Ídem \\
\hline Incomprensión en clase & Clase, 11 & Ídem \\
\hline No se conoce el camino & Camino, 12 & Ídem \\
\hline Desconfianza en sí mismo & Confi, 13 & Idem \\
\hline Fallo en cálculos & Calcu, 14 & Ídem \\
\hline
\end{tabular}

Curso

Fecha

- ¿Cómo definitías to que es un problema en física y química?

- ¿Qué es más difícil, la teoría o los problemas?

- ¿Cuáles son las dificultades que encuentras? Califícalas todas entre 1 y 5 (1=las menos importantes, 5=las más importantes).

II Se sabe la teoría pero no se sabe aplicarla al problema.

I I No se entiende lo que pide el problema.

[I Hay falta de conocimientos (no se sabe lo necesario).

[ I Falla la memoria (se olvida lo que ya se sabía).

[ I Falta de trabajo y estudio.

[ ] Falta de interés.

II Son demasiado complicados.

[ I No se entienden los problemas que se hacen en clase.

I I No se tiene claro el camino para resolverlos.

[ I Antes de empezar ya se sabe que no va a salir bien.

[ ] Fatlo en los cálculos matemáticos y operaciones.

- Otras posibles dificultades que se te ocurran: 